\title{
An infant presenting with Kawasaki disease following immunization for influenza: A case report
}

\author{
SEUNG WOO JEONG ${ }^{1}$, DO HEE KIM ${ }^{1}$, MI YOUNG HAN ${ }^{2}$, SUNG-HO CHA $^{2}$ and KYUNG LIM YOON ${ }^{1}$ \\ ${ }^{1}$ Department of Pediatrics, Kyung Hee University Hospital at Gangdong, Kyung Hee University \\ School of Medicine, Seoul 05278; ${ }^{2}$ Department of Pediatrics, Kyung Hee University Hospital, \\ Kyung Hee University School of Medicine, Seoul 02447, Republic of Korea
}

Received March 7, 2017; Accepted June 12, 2017

DOI: $10.3892 /$ br.2018.1043

\begin{abstract}
Kawasaki disease (KD) is a childhood vascular disorder of unknown etiology. Concerns have recently been raised regarding vaccinations as a potential risk factor for KD. In addition, various forms of vasculitis have been reported as adverse events following administration after various vaccines. Patients exhibiting post vaccination KD have previously been described; however, thus far, to the best of our knowledge, only one patient exhibiting post influenza vaccination KD has been reported in Japan. The present study describes a case of KD $24 \mathrm{~h}$ after immunization with influenza in an infant (age, 18 months) following 6 days of high fever, a body rash that had persisted for 2 days and nonsuppurative bilateral conjunctivitis. To the best of the authors' knowledge, this is the first reported case in Korea and the present study reviews various recent studies regarding vasculitis following vaccination and the causal association between them.
\end{abstract}

\section{Introduction}

Kawasaki disease (KD) is one of the most common vasculitides of childhood (1). The cause of KD remains unknown; however, it is hypothesized that the immune system is activated by infectious or environmental triggers in genetically susceptible hosts (1). Previous investigations into vasculitis and KD have reported them as side effects of various vaccinations (2-4). Bonetto et al (5) reviewed the literature from January 1994 to June 2014. Although the majority of the larger, higher quality studies identified no causal association between vaccination and subsequent development of vasculitis, including various studies on KD and Henoch-Schönlein purpura (HSP), the influenza vaccination was ranked first in terms of the number

Correspondence to: Dr Kyung Lim Yoon, Department of Pediatrics, Kyung Hee University Hospital at Gangdong, Kyung Hee University School of Medicine, 892 Dongnam-ro, Gangdong-gu, Seoul 05278, Republic of Korea

E-mail: ykr3215@khnmc.or.kr

Key words: Kawasaki disease, influenza vaccination, vasculitis of published articles regarding vasculitis vaccine association (according to vaccine type) and KD was third in terms of the number of published articles regarding vasculitis vaccine association (according to vasculitis type) (5). Cases were reported in which children had received vaccinations, such as those for yellow fever and hepatitis B, and developed $\operatorname{KD}(2,3)$. However, a report in 2015 on KD that occurred following influenza vaccination was the only case reported in Japan (4). The present study describes the first case of KD following immunization for influenza in South Korea and reviews various studies regarding vasculitis following vaccination and the causal association between them.

\section{Case report}

A male infant, aged 18 months, was admitted to Kyung Hee University Hospital at Gangdong (Seoul, South Korea; 12 October 2015) for evaluation due to a high-grade fever that had started 5 days prior to the visit. The infant had been immunized for influenza $24 \mathrm{~h}$ before the onset of fever. $\mathrm{He}$ was admitted as he had experienced 6 days of high fever up to $40.0^{\circ} \mathrm{C}$, a body rash that had persisted for 2 days and nonsuppurative bilateral conjunctivitis. Informed consent for the publication of this case report was waived as the infant's next of kin could not be contacted despite numerous attempts to do so.

The body temperature of the infant at the time of admittance was $39.6^{\circ} \mathrm{C}$. The physical examination demonstrated bilateral conjunctival injection, cracked red lips, strawberry tongue, erythema of the trunk and the Bacille Calmette-Guérin inoculation site, and erythema and bilateral edema of the hands. However, the cervical lymph nodes appeared normal. The peripheral blood test exhibited leukocytosis (white blood cell count, 12,110 cells/ $\mu 1$ ), an elevated level of C-reactive protein $(15.1 \mathrm{mg} / \mathrm{dl})$ and an elevated quantity of brain natriuretic peptide (403 pg/ml; Table I). Echocardiography demonstrated that the size of the coronary artery was within the normal range, although the left ventricular ejection fraction was somewhat reduced to $50-55 \%$. Based on these findings, KD treatment was initiated with intravenous immunoglobulin (IVIG; $2 \mathrm{~g} / \mathrm{kg} /$ dose) for 1 day and oral administration of aspirin ( $50 \mathrm{mg} / \mathrm{kg} /$ day). After the end of the IVIG treatment, the fever temporarily improved. However, the infant was injected with 
Table I. Laboratory data during the illness.

Days after fever onset

\begin{tabular}{lccc}
\cline { 2 - 4 } Parameter & 6 & 9 & 13 \\
\hline White blood cells (cells/ $\mu \mathrm{l})$ & $12.11 \times 10^{3}$ & $8.33 \times 10^{3}$ & $8.62 \times 10^{3}$ \\
Neutrophils (\%) & 65 & 40 & 26 \\
Hemoglobin $(\mathrm{g} / \mathrm{dl})$ & 10.4 & 10.9 & 10.6 \\
Hematocrit $(\%)$ & 31.4 & 34 & 33.4 \\
Blood platelets (platelets/ $\mu \mathrm{l})$ & $344 \times 10^{3}$ & $377 \times 10^{3}$ & $625 \times 10^{3}$ \\
Erythrocyte sedimentation rate $(\mathrm{mm} / \mathrm{h})$ & 70 & $>120$ & 110 \\
C-reactive protein $(\mathrm{mg} / \mathrm{dl})$ & 15.1 & 8.6 & 1.2 \\
Aspartate aminotransferase (IU/l) & 49 & 36 & 35 \\
Alanine aminotransferase (IU/l) & 39 & 21 & 12 \\
Brain natriuretic peptide $(\mathrm{pg} / \mathrm{ml})$ & 403 & 172 & 23
\end{tabular}

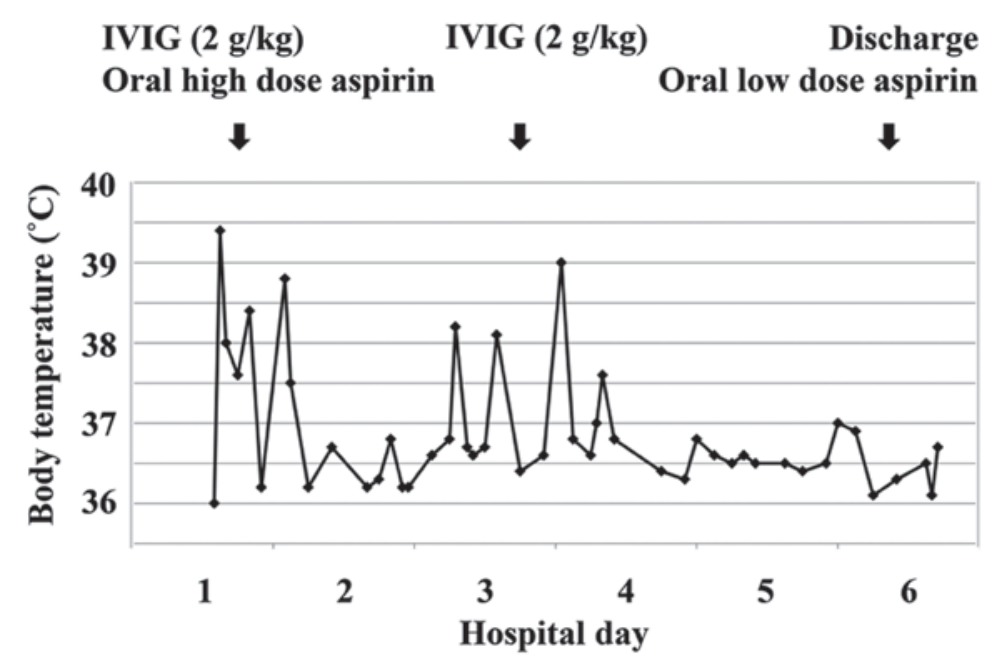

Figure 1. Clinical progress during hospitalization. IVIG, intravenous immunoglobulin.

additional IVIG as a result of a relapse of fever. The second IVIG treatment resulted in the rapid improvement of the KD symptoms, decreased white blood cell count $(8,330$ cells $/ \mu \mathrm{l})$, level of C-reactive protein $(8.6 \mathrm{mg} / \mathrm{dl})$, quantity of brain natriuretic peptide $(172 \mathrm{pg} / \mathrm{ml})$ and recovered left ventricular ejection fraction $(60 \%)$. Therefore, the dose of oral aspirin was reduced to $5 \mathrm{mg} / \mathrm{kg} /$ day (Fig. 1). The elevated blood platelet count $(625,000$ platelets $/ \mu 1)$ was then confirmed 7 days after the onset of the disease (Table I).

\section{Discussion}

Concerns have been raised regarding vaccinations being a potential risk factor leading to KD (6). Various types of vasculitis have been observed and reported in temporal association with the administration of certain vaccines (5).

Abrams et al (6) collected and analyzed data of children surveyed from 1996 to 2006 from the Vaccine Safety Datalink (https://www.cdc.gov/vaccinesafety/ensuringsafety/monitoring/vsd/publications.html), and investigated the relevance of childhood vaccines and KD. However, the authors found no evidence that any of the vaccinations increased the risk of $\mathrm{KD}(6)$.
Conversely, Zafrir et al (7) demonstrated a possible link between the influenza vaccination and autoimmunity, though the mechanism underlying the development of influenza vaccine-induced vasculitides remains unknown. As an activated immune system following infectious or environmental stimulation contributes to the development of $\mathrm{KD}$, the influenza vaccination may serve as a trigger for the development of KD (4).

Bonetto et al (5) reviewed the literature from 1994 to 2014 and reported that influenza vaccination was ranked first in the number of published articles on vasculitis vaccine association (according to vaccine type) and KD was placed third in the number of published articles on vasculitis vaccine association (according to vasculitis type) (5). Despite the large number of reports, as well as published case reports and case series, suggesting an association between vaccination and vasculitis, the few observational and clinical trials investigating associations between various vaccines and vasculitis subtypes have failed to confirm such an association (5). However, there is increasing evidence establishing various types of infections as potential triggers or causes of different types of vasculitis (5). Hoffman et al (8) reported a potential association between 
the hepatitis B (HBV) and hepatitis C (HCV) infections and polyarteritis nodosa (PAN) and cryoglobulinemic vasculitis. In France, a successful campaign for vaccination against HBV was followed by a decrease in the incidence of PAN (9). Furthermore, emerging evidence has led to the hypothesis that there is a potential role of infection in KD as well (10). Therefore, the potential of vaccination-induced vasculitis requires consideration in the context of the decreased risk of infection-induced vasculitis (5).

Post-HBV, post-yellow fever and post-influenza vaccination KD have previously been reported (2-4). However, a 2015 report on KD that was performed following vaccination for influenza was, to the best of our knowledge, the only case reported in Japan (4). The present study describes the first case of KD following immunization for influenza in South Korea and reviews previous studies on vasculitis following vaccination and the causal association between them.

In conclusion, it may be hypothesized that influenza vaccination is a trigger of $\mathrm{KD}$ when considering the timing of vaccination and onset of KD, although the present study was unable to demonstrate a direct relevance of the influenza vaccine to the onset of KD.

\section{Acknowledgements}

Not applicable.

\section{Funding}

No funding was received.

\section{Availability of data and materials}

The analyzed data sets generated during the study are available from the corresponding author on reasonable request.

\section{Authors' contributions}

JSW analyzed and interpreted the patient data including the laboratory finding and the pattern of fever. KDH made tables and graph including patient's information. HMY searched and analyzed references. $\mathrm{CSH}$ reviewed and revised about the manuscript. YKL performed an echocardiogram and was a major contributor in writing the manuscript. The final version of the manuscript has been read and approved by all authors.

\section{Ethics approval and consent to participate}

Informed consent for the publication of this case report was waived as the infant's next of kin could not be contacted despite numerous attempts to do so.

\section{Consent for publication}

Not applicable.

\section{Competing interests}

The authors declare that they have no competing interests.

\section{References}

1. Sundel RP and Petty RE: Kasawaki disease. In: Textbook of Pediatric Rheumatology. 6th edition. Classidy JT, Laxer RM, Petty RE and Lindsley CB (eds). Elsevier Saunders, Philadelphia, pp505-520, 2011

2. Miron D, Fink D and Hashkes PJ: Kawasaki disease in an infant following immunisation with hepatitis B vaccine. Clin Rheumatol 22: 461-463, 2003.

3. Schmöeller D, Keiserman MW, Staub HL, Velho FP and de Fátima Grohe M: Yellow fever vaccination and Kawasaki disease. Pediatr Infect Dis J 28: 1037-1038, 2009.

4. Shimada S, Watanabe T and Sato S: A Patient with Kawasaki Disease Following Influenza Vaccinations. Pediatr Infect Dis J 34: 913, 2015.

5. Bonetto C, Trotta F, Felicetti P, Alarcón GS, Santuccio C, Bachtiar NS, Pernus YB, Chandler R, Girolomoni G, Hadden RDM, et al: Vasculitis as an adverse event following immunization-Systematic literature review. Vaccine, 2015.

6. Abrams JY, Weintraub ES, Baggs JM, McCarthy NL, Schonberger LB, Lee GM, Klein NP, Belongia EA, Jackson ML, Naleway AL, et al: Childhood vaccines and Kawasaki disease, Vaccine Safety Datalink, 1996-2006. Vaccine 33: 382-387, 2015.

7. Zafrir Y, Agmon-Levin N and Shoenfeld Y: Post-influenza vaccination vasculitides: A possible new entity. J Clin Rheumatol 15: 269-270, 2009.

8. Hoffman GS and Calabrese LH: Vasculitis: Determinants of disease patterns. Nat Rev Rheumatol 10: 454-462, 2014

9. Guillevin L: Infections in vasculitis. Best Pract Res Clin Rheumatol 27: 19-31, 2013.

10. Principi N, Rigante D and Esposito S: The role of infection in Kawasaki syndrome. J Infect 67: 1-10, 2013. 